\title{
CONTRIBUTIONS TO THE KNOWLEDGE ON STAPHYLINIDS (COLEOPTERA: STAPHYLINIDAE) IN SOME RIPARIAN ECOSYSTEMS OF SOUTH-EASTERN ROMANIA
}

\author{
MELANIA STAN
}

\begin{abstract}
The diversity of the staphylinid fauna is investigated in some riparian ecosystems along rivers of south-east Romania: the Danube, Prut, Siret, Buzău. 94 staphylinid species and subspecies were identified from 23 investigated sites. Thecturota marchii (Dodero) is a new record for the Romanian fauna. Leptobium dimidiatum (Grideli), a rare species, is recorded from a new site, the second record from Romania.
\end{abstract}

Résumé. On présente la diversité de la faune de staphylinides dans quelques écosystèmes ripariens qui se trouvent le long des rivières du sud-est de la Roumanie: Danube, Prut, Siret, Buzău. 94 espèces et sous-espèces de staphylinides y ont été trouvées, en 23 sites. Theucturota marchii (Dodero) est signalée pour la première fois en Roumanie. Leptobium dimidiatum (Grideli), une espèce rare, est signalée dans un nouveau site, le deuxième sur le territoire roumain. Pour chaque espèce on présente le site où elle a été trouvée, la date, la nombre d'exemplaires (pour la plupart le sexe), legit. Sur la base des observations faites sur le terrain on offre une brève référence sur la caractéristique écologique des espèces.

Key words: Staphylinidae, riparian ecosystems, faunistics.

\section{INTRODUCTION}

The hydrobiologic regime represents the most important control element for the existence, characteristics and maintaining of the wetland types and of their characteristic processes. Riparian areas are very important for the delimitation of the ecosystems, but especially in the specific functions which they have within the ecosystem complexes: flooding control, protection against erosion, supplying/ discharging of the underground waters, nutrient retention, biomass export, protection against storms, water transportation, stabilization of the microclimate.

Flooding areas which are in a natural regime of flooding are characterized by high biodiversity. Staphylinids live in such kind of habitats - most of the species are hygrophilous, some of them are stenotopic and biological indicators.

Some riparian ecosystems studied in order to find out the diversity of the staphylinids are protected areas, designated at local or national level: The Small Wetland of Brăila Natural Park, the Low Floodplain of the Lower Prut Natural Park. To them, there are superposed Sites of Community Importance (SCI), established according to the presence of some plant and animal species, as well as of certain types of habitats listed in the annex of the Habitats Directive 92/43/EEC+AA2003/ACT: 
the Small Wetland of Brăila (ROSCI0006), Low Floodplain of Prut (ROSCI0105). Lower Siret Floodplain, another investigated area, is an Area of Avifaunal Special Protection (SPA). SPA are established based on some bird species listed in the Bird Directive 78/409/EEC+AA2003/ACT of the European Union.

\section{MATERIAL AND METHODS}

The material was sampled in some riparian ecosystems along some rivers of south-east Romania, during different periods: 1994-1995 (Small Wetland of Brăila Natural Park), 2002-2007, 2010 (Buzău valley), 2004 and 2008 (Danube isles), 2009 (Siret floodplain) and 2010 (Low Floodplain of the Lower Prut Natural Park).

A brief description of the sampling places is presented below:

The Small Wetland of Brăila Natural Park - a wetland of international importance, is situated in the Danube floodplain, upstream of the Danube Delta Biosphere Reservation, and includes terrestrial and aquatic ecosystems whose configuration depends on the current annual dynamics of the Danube flow, particularly on the amplitude and the duration of the seasonal high floods. Terrestrial ecosystems cover over $60 \%$ of the surface and are represented by forests, meadows and bushes. Autochthonous elements of the forest resources are represented by the willow and poplar forests, both of which are, unfortunately, in decline. There are natural mixed forests which form the link between the compact willow forests which surround the pools and the depression areas. In the areas where the willow forests were cut there are plantations with American and Italian poplar. Aquatic ecosystems are represented by permanent pools, temporary pools (which gradually evolve into aquatic ecosystems of the marshy type or marshy area and finally dry up), marshes, marshy areas, permanent and temporary channels.

Lower Prut Natural Park - a prolongation of the Danube Delta Biosphere Reserve is a wetland area of avifaunal interest both for Romania as well as for the Southeast Europe.

Within the park area, a multitude of habitats was identified: mixed riparian forests, riverside coppices, low altitude wetland meadows, forest skirts with high bushes, muddy bank rivers, eutrophic natural lakes, oligotrophic to mesotrophic still waters, dystrophic lakes and ponds.

Lower Floodplain of Siret River is characterized by floodplain vegetation: riparian galleries and bushes, salty marshes, riverside coppices with Salix alba and Populus alba, oligotrophic to mesotrophic still waters.

The staphylinid fauna from some Danube islets (Giurgiu and Călăraşi sectors) has been addressed in two papers (Stan, 2005, 2009). In the present paper, I report the species which have to be added to those previously published, the specimens being identified by László Ádám (Hungarian Natural History Museum, Budapest). 
The floodplain area of the Buzău valley is characterized by an intense activity of exploitation of the river bed for sand and gravel. Sampling was conducted in the few areas without anthropic impact. Most of the data are from oak, elm, hornbeam forests.

Collecting sites are presented in fig. 1:

- Small Wetland of Brăila Natural Park: Small Island of Brăila, Gura Gârluţei; Chirchineţu fish farm (an ex-fish breeding place), south of Small Island of Brăila; Fundu Mare Island; Hogioaia Channel-entry in Stan's Marsh.

- Lower Prut Natural Park: Vădeni, Cavadineşti, Maţa-Rădeanu Complex, (N: 46 $04.218^{\prime}$, E: $\left.028^{\circ} 05.315^{\prime}\right)$, bank of the Prut River, flooded area in summer; Rogojeni, Pochina Lake, wetland area with Salix spp. and Rubus

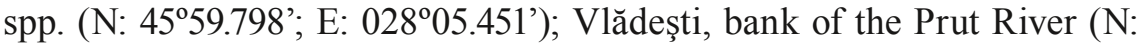

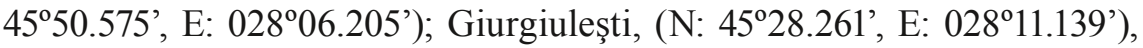
area with temporary pools, a result of the summer flooding.

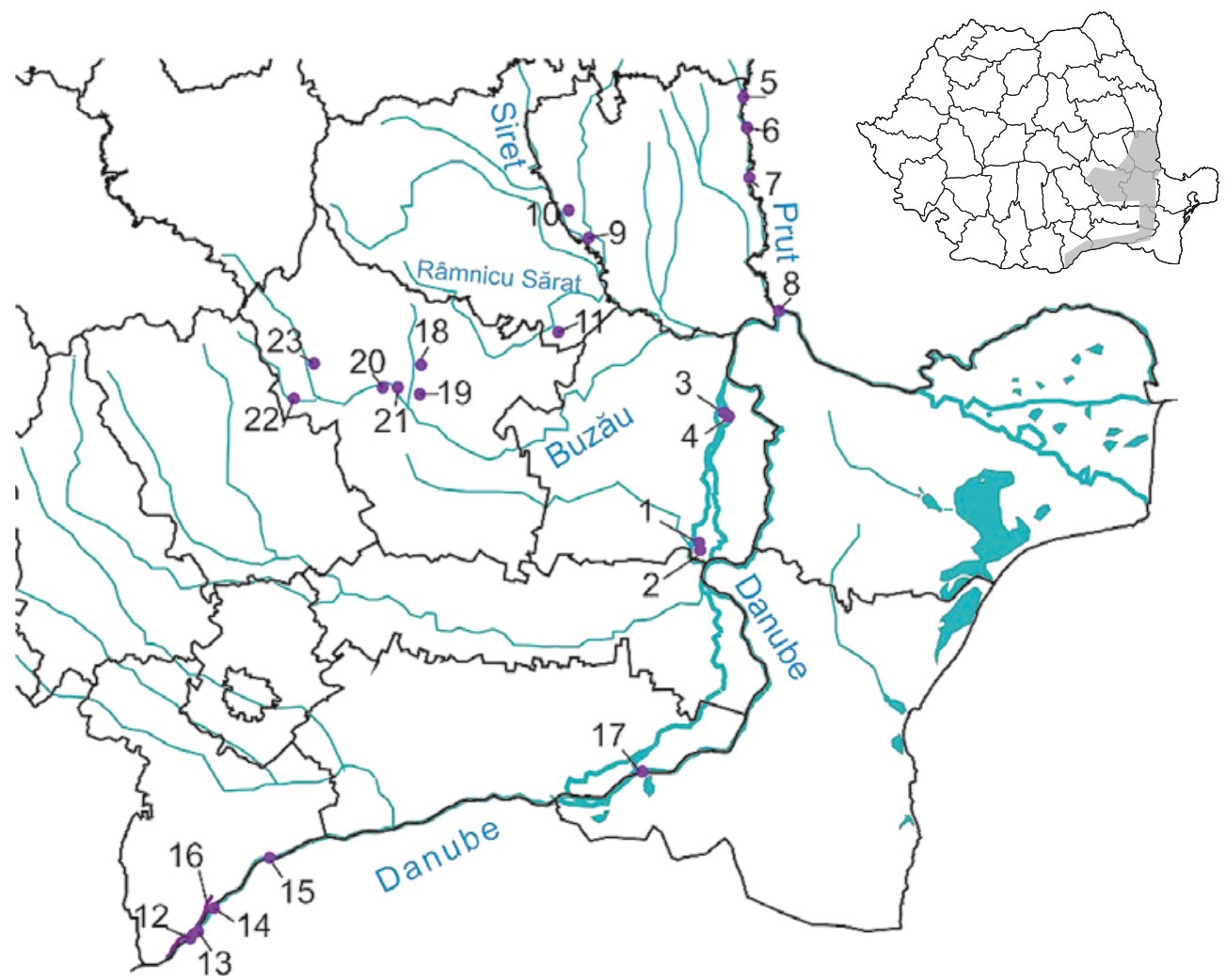

Fig. 1 - The map of collecting sites from south-east of Romania. 1 - Gura Gârluței; 2 - Chirchineţu fish farm (ex-fish breeding place); 3 - Fundu Mare Island; 4 - Hogioaia Channel-entry in Stan's Marsh; 5 Vădeni; 6 - Rogojeni; 7 - Vlădeşti; 8 - Giurgiuleşti; 9 - Salcia; 10 - Movileni; 11 - Ciorăşti; 12 - Cama Islet, 13 - Dinu Islet; 14 - Slobozia Islet; 15 - Lung Islet; 16 - dike area (508 - 521 km); 17 - Turcescu Islet; 18 - Izvorul Dulce; 19 - Cernăteşti; 20 - Unguriu; 21 - Berca; 22 - Bâscenii de Sus; 23 - Valea Seaca. 
- Siret Floodplain: Salcia (N: 45040.606', E: 027²6.701', Umbrăreşti commune), sandy bank of Siret River; Movileni (N: 45 43.619'; E:

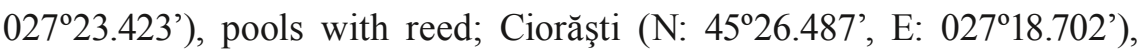
bank of Râmnicu Sărat River.

- Giurgiu Danube sector: islets Cama (km 510), Dinu (km 507), Slobozia $(\mathrm{km} \mathrm{500)}$, Lung ( $\mathrm{km} \mathrm{468)}$ and the dam area: Malu ( $\mathrm{km} \mathrm{508),} \mathrm{Vedea}(\mathrm{km}$ 517), Cetăţuia (km 516), Pietroşani (km 521); Călăraşi Danube sector Turcescu Islet (km 344).

- Basin of the Buzău River: valley of the Slănic River - Izvorul Dulce (Beceni commune) and Cernăteşti; valley of the Buzău River - Unguriu (Măgura commune), Berca, Bâscenii de Sus (Calvini commune) - Chiojdului valley (tributary of Buzău, N: 45¹5.094', E: 026²16.964'), Valea Seaca (Pătârlagele commune, N: $45^{\circ} 16.653^{\prime}$, E: $\left.026^{\circ} 21.605^{\prime}\right)$ - bank of the river.

Abbreviations.

In the faunistic list, the collectors' names from "Grigore Antipa" National Museum of Natural History, Bucharest and collaborators of the Museum are abbreviated as follows: C.C. - Ciubuc Constantin; P.C. - Pârvu Corneliu; S.E. - Schneider Eckbert; S.M. - Stan Melania; U.V. - Ungureanu Viorel; ex. (s) - exemplar (s).

The rove beetles were collected using the pitfall traps (for the material from Small Island of Brăila Natural Park, during the period 15.06-30.12.1994, replaced every two weeks. The dates mentioned in the paper represent the day when the traps were taken), aspirators, light traps with a mercury vapour bulb (basin of the Buzău River), by the flotation method, direct collecting from the ground, collecting from mushrooms, cattle, horse and pig dung.

The specimens are preserved in the Coleoptera Collection of "Grigore Antipa" National Museum of Natural History (Bucharest).

\section{RESULTS AND DISCUSSIONS}

The paper presents 94 staphylinid species and subspecies, collected from some riparian ecosystems associated with some rivers in southeastern Romania: the Danube, Prut, Siret, Buzău.

The identified staphylinid species are grouped according to subfamilies, and listed alphabetically within subfamilies. Species of 7 subfamilies were identified: Pselaphinae (1), Aleocharinae (30), Oxytelinae (19), Euaestethinae (1), Steninae (6), Paederinae (11) and Staphylininae (26). For each species, the collecting site, date, number of specimens (sex, for most of them), and the collector are specified. The species marked with an asterisk were identified by László Ádám (Budapest, Hungary). 
Subfamily Pselaphinae Latreille, 1802

Rybaxis longicornis (Leach, 1817)

Examined material: 2 exs., Fundu Mare Island, temporarily flooded, 30.10.1994, leg. C.C.

Subfamily Aleocharinae Fleming, 1821

Acrotona muscorum* (Brisout de Barneville, 1860)

Examined material: 1 ex., Turcescu Islet, 26.05.2008, leg. S.M.

Aleochara brevipennis Gravenhorst, 1806

Examined material: 1 ex., Hogioaia Channel-entry in Stan's Marsh, frequently flooded, 3.09.1994, leg. C.C.

Aleochara curtula (Goeze, 1777)

Examined material: 1 ex., Hogioaia Channel-entry in Stan's Marsh, rarely flooded, 30.07.1994; 1 ex., Gura Gârluţei, rarely flooded, 4.10.1994; 1 đo, 1 + , Fundu Mare Island, rarely flooded, 4.10.1994, leg. C.C.; 1 ex., Izvorul Dulce, 2.05.2002, leg. U.V. Aleochara haematoptera Kraatz, 1858

Examined material: 2 exs., Chirchinețu fish farm, rarely flooded, 16.08.1994; 2 exs., Gura Gârluţei, frequently flooded, 16.09.1994; 42 exs., the same place, rarely flooded area, 4.10.1994; 2 O, the same place, frequently flooded 12.10.1994; 11 exs., Fundu Mare Island, rarely flooded and 7 exs. the same place in a frequently flooded area, 4.10.1994, leg. C.C.

Aleochara intricata Mannerheim, 1830

Examined material: 1 ex., Izvorul Dulce, 30.04 .2005 (on carcasses) and 3 exs., the same site, 3.05.2005, on fresh dung, bed of the Slănic River, leg. U.V.

Aloconota gregaria (Erichson, 1839)

Examined material: 1 +, bank of the Râmnicu Sărat River, Ciorăşti area, 27.05.2009, leg. S.M.

Amischa analis (Gravenhorst, 1802)

Examined material: 1 , , Hogioaia Channel-entry in Stan's Marsh, rarely flooded, 15.07.1994, leg. C.C.

Amischa bifoveolata (Mannerheim, 1830)

Examined material: 1 đ̂, Hogioaia Channel-entry in Stan's Marsh, 15.11.1994, rarely flooded; 1 ð’, Gura Gârluţei, frequently flooded, 29.11.1994, leg. C.C.

Amischa forcipata Mulsant \& Rey, 1873

Examined material: 1 กे, Hogioaia Channel-entry in Stan's Marsh, frequently flooded, 3.10.1994; 2 \%, the same sampling place, frequently flooded area, 15.11.1994 and $1 \stackrel{\partial}{\partial} 1$ q the same place, 28.11.1994, leg. C.C.

Atheta atramentaria (Gyllenhal, 1810)

Examined material: 3 exs., Izvorul Dulce, 3.05.2005, on fresh dung, bed of Slănic River, leg. U.V. 
Atheta fungi (Gravenhorst, 1806)

Examined material: 1 ex., Chirchineţu fish farm, rarely flooded, 16.08.1994; 1 ô, 1

, Hogioaia Channel-entry in Stan's Marsh (frequently flooded), 15.11.1994; 1 o, 1

+, Gura Gârluţei, frequently flooded, 29.11.1994, leg. C.C.

Atheta orbata* (Erichson, 1837)

Examined material: 2 exs., Turcescu Islet, 26.05.2008, leg. S.M.

Atheta laticollis (Stephens, 1832)

Examined material: 1 + , Gura Gârluţei, frequently flooded, 16.09.1994; 1 ex., Fundu Mare Island, frequently flooded, 4.10 .1994 and 1 q, 18.10.1994; 1 + , Hogioaia

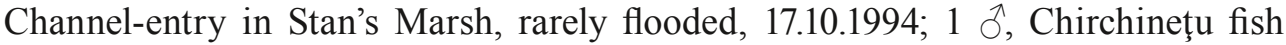
farm, rarely flooded, 30.10.1994.

Atheta melanaria (Mannerheim, 1830)

Examined material: 1 ex., Izvorul Dulce, 3.05.2005, on fresh dung, bed of Slănic River, leg. U.V.

Atheta xanthopus* (Thomson, 1856)

Examined material: 1 ex., Cama Islet, 7-16.06.2004, leg. S.M.

Brachyusa concolor (Erichson, 1839)

Examined material: 7 Ō, 4 क, Vădeni, 29.09.2010, bank of Prut River, flooded area in summer, leg. S.M.

Gyrophaena lucidula Erichson, 1837

Examined material: $16 \hat{\jmath}, 12$ ๆ, Giurgiuleşti, 30.09.2010, area with temporary pools, a result of the summer floodings, in mushrooms, leg. S.M.

Liogluta microptera Thomson, 1867

Examined material: 1 ex., Gura Gârluţei, rarely flooded, 4.10.1994, leg. C.C.

Meotica marchica* Benick, 1954

Examined material: 1 ô, Hogioaia Channel-entry in Stan's Marsh, frequently flooded area, 15.07.1994; 1 o, the same place, 15.11.1994, leg. C.C.

Myllaena intermedia Erichson, 1837

Examined material: 1 ô, Giurgiulești, 30.09.2010, area with temporary pools, a result of the summer floodings, leg. S.M.

Oligota pumilio Kiesenwetter, 1858

Examined material: 1 q, Gura Gârluţei, temporarily flooded, 3.10.1994, leg. C.C.

Oxypoda filiformis* Redtenbacher, 1849

Examined material: 1 q, Gura Gârluţei, frequently flooded, 29.11.1994; 1 ex., Turcescu Islet, 26.05.2008, leg. S.M.

Oxypoda haemorrhoa (Mannerheim, 1830)

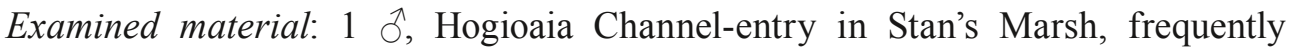
flooded, 28.11.1994, leg. C.C.

Oxypoda longipes* Mulsant \& Rey, 1861

Examined material: 1 ex., Pietroşani, 28.04.2004, leg. P.C. 
Oxypoda opaca (Gravenhorst, 1802)

Examined material: 1 ô, Gura Gârluţei, rarely flooded, 15.11.1994; 1 ô, Hogioaia Channel-entry in Stan's Marsh, 15.11.1994, leg. C.C.

Oxypoda vicina* Kraatz, 1858

Examined material: 1 ex., Cetăţuia, 26.04.2004, leg. S.M.

Tachyusa coarctata Erichson, 1837

Examined material: 1 ex., Salcia, sandy bank of the Siret River, 27.05.2009; 1 ex., Ciorăşti, the bank of the Râmnicu Sărat River, 27.05.2009, leg. S.M.

Tachyusa concinna* Heer, 1839

Examined material: 1 ex., Slobozia Islet, 21.06.2004, leg. S.M.

Tachyusa objecta Mulsant \& Rey, 1870

Examined material: 2 exs., Hogioaia Channel-entry in Stan's Marsh, rarely flooded, 15.07.1994; 1 §ै, Gura Gârluței, frequently flooded, 16.09.1994; 3 exs., the same place, frequently flooded, 29.11.1994, leg. C.C.; 5 exs., Ciorăşti, the bank of the Râmnicu Sărat River, 27.05.2009, leg. S.M.; 3 exs., Vădeni, 29.09.2010, bank of Prut River, area flooded in summer, leg. S.M.; 1 \&, 1 $\hat{\text { }}$, Rogojeni, Pochina Lake, wetland with Salix spp. and Rubus spp., 29.09.2010, leg. S.M.; 6 exs., Vlădeşti, bank of Prut River, 29.09.2010, leg. S.M.

Thecturota marchii (Dodero, 1922) (Fig. 2 A-D)

Examined material: 1 q, Fundu Mare Island, frequently flooded, 4.10.1994, leg. C.C. First record from Romania.

Remarks: Thecturota is represented by two species in Europe, Thecturota williamsi (Bernhauer), known only from the United Kingdom, and T. marchii (Dodero) recorded from Austria, Czech Republic, Denmark, Estonia, Finland, France, United Kingdom, Germany, Italy, Norway, Sweden, Switzerland (Smetana, 2004). According to Horion (1967) the species is distributed in North Europe (southern part), Central and South Europe, its range expanding, and occurs in rotting plant material (compost) and debris.

Subfamily Oxytelinae Fleming, 1821

Anotylus affinis (Czwalina, 1871)

Examined material: $4 \hat{\sigma}$, Hogioaia Channel-entry in Stan's Marsh, temporarily flooded, 1.08.1994, leg. C.C.; 1 đ, Gura Gârluţei, frequently flooded, 16.09.1994; 5 exs., Izvorul Dulce, 3.05.2005, on cattle fresh dung, bed of Slănic River, leg. U.V.

Anotylus insecatus (Gravenhorst, 1806)

Examined material: 1 ex., Izvorul Dulce, 10.04.2002, leg. U.V.

Anotylus intricatus (Erichson, 1840)

Examined material: 23 exs., Izvorul Dulce, 3.05.2005, on cattle fresh dung, bed of Slănic River, leg. U.V.

Anotylus nitidulus (Gravenhorst, 1802)

Examined material: 1 +, bank of Râmnicu Sărat River, Ciorăşti area, 27.05.2009, cattle dung, leg. S.M. 


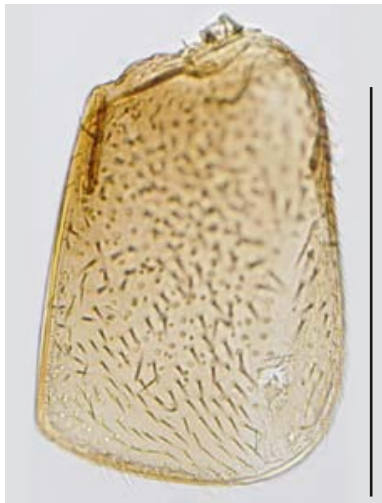

A

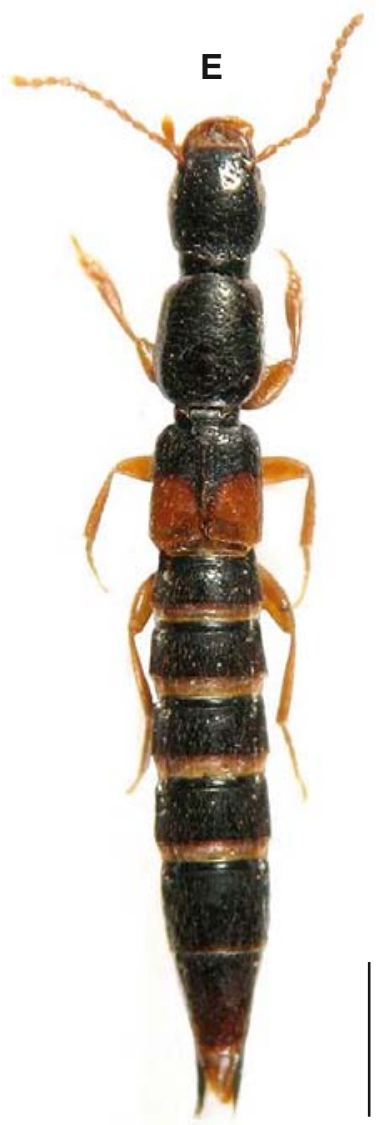

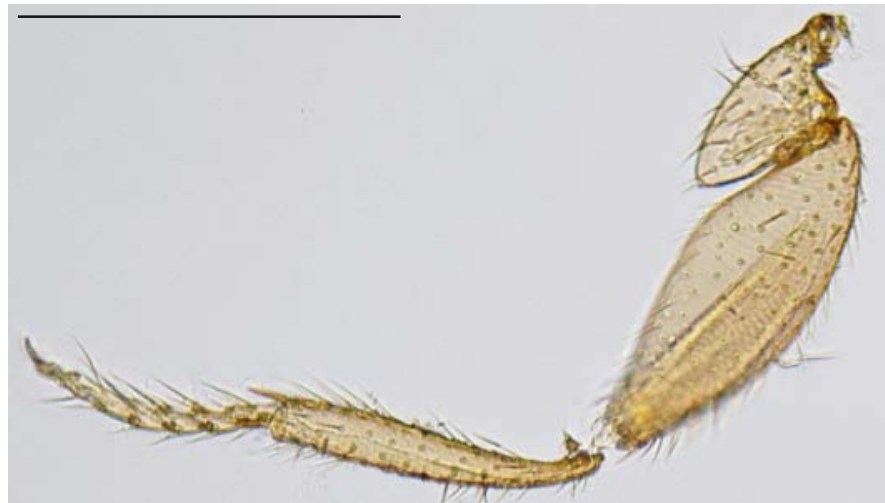

B

C
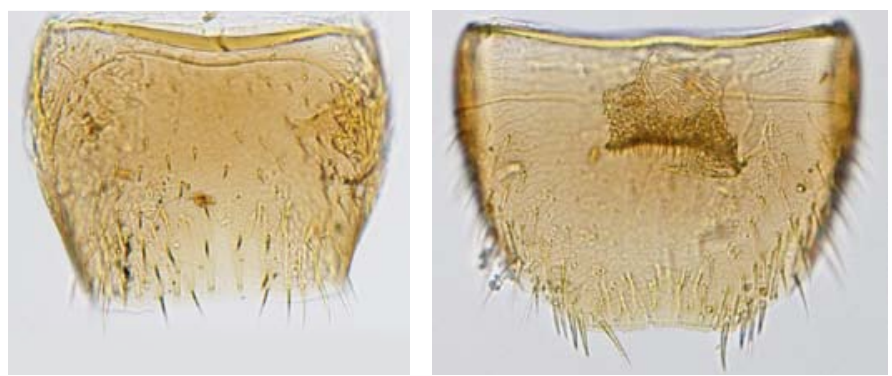

D

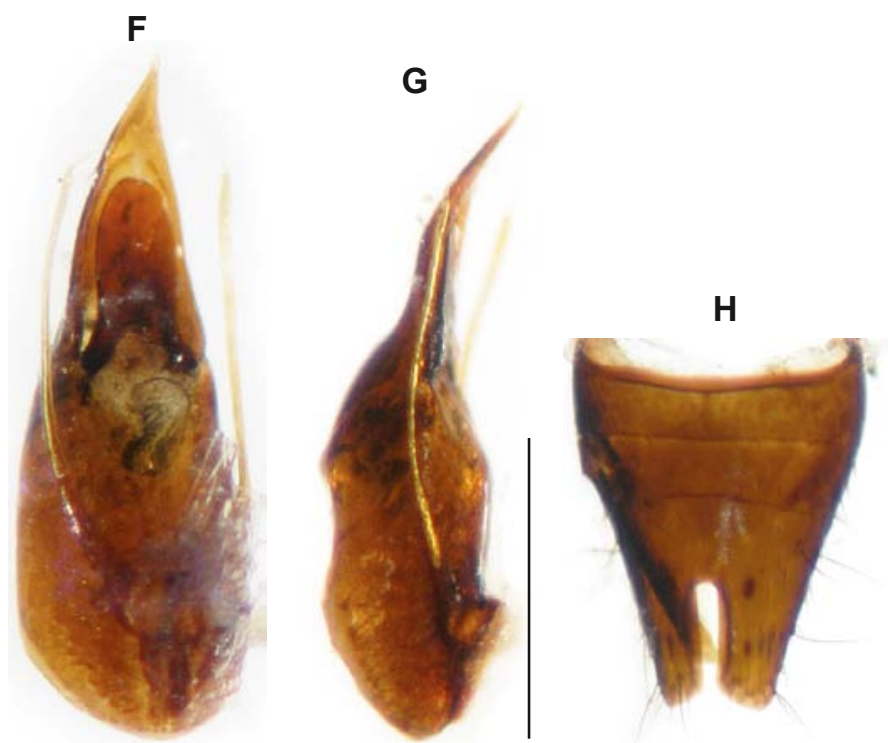

Fig. 2 - Thecturota marchii (Dodero): A - left elytron; B - hind leg; C - tergit VIII (internal view); D - sternite VIII (internal view). Leptobium dimidiatum (Gridelli): E - habitus; F - aedeagus (ventral view); G - aedeagus (lateral view); H - male sternite VIII. Scale bars: $1 \mathrm{~mm}$ (E), $0.25 \mathrm{~mm}$ (A-D, F-H). 
Anotylus pumilus (Erichson, 1839)

Examined material: 6 exs., Izvorul Dulce, 3.05.2005, on cattle fresh dung, bed of Slănic River, leg. U.V.

\section{Anotylus tetracarinatus (Block, 1799)}

Examined material: 5 exs., Izvorul Dulce, 3.05.2005, on cattle fresh dung, bed of Slănic River, leg. U.V.

Carpelimus corticinus (Gravenhorst, 1806)

Examined material: 1 ex., Hogioaia Channel-entry in Stan's Marsh, frequently flooded, 15.11.1994, leg. C.C.; 1 ex., Izvorul Dulce, 4.08.2005, leg. U.V.

Carpelimus exiguus (Erichson, 1839)

Examined material: 1 ex., Fundu Mare Island, frequently flooded, 4.10.1994; 1 ex., Gura Gârluței, temporarily flooded, 3.10.1994; 1 ex., Chirchineţu fish farm, temporarily flooded, 30.10.1994; 2 exs., Hogioaia Channel-entry in Stan's Marsh, frequently flooded, 15.11.1994, leg. C.C.; 2 exs., Salcia, sandy bank of Siret River, 27.05.2009, leg. S.M.; 2 exs., Vădeni, 29.09.2010, bank of Prut River, area flooded in summer, leg. S.M.; 2 exs., Rogojeni, Pochina Lake, wetland with Salix sp. and Rubus sp., 29.09.2010, leg. S.M.; 1 ex., Vlădeşti, bank of Prut River, 29.09.2010, leg. S.M.; 1 ex., Giurgiuleşti, 30.09.2010, area with temporary pools, a result of the summer flooding, leg. S.M.

Carpelimus gracilis (Mannerheim, 1830)

Examined material: 1 ex., Hogioaia Channel-entry in Stan's Marsh, frequently flooded, 3.10.1994; 1 ex., Fundu Mare Island, frequently flooded, 4.10.1994; 1 ex., Gura Gârluţei, frequently flooded, 29.11.1994, leg. C.C.

Carpelimus impressus (Lacordaire, 1835)

Examined material: 1 Ô, Vădeni, 29.09.2010, bank of Prut River, area flooded in summer, leg. S.M.; 1 ô, Lung Islet, 22.06.2004, leg. P.C.

Carpelimus obesus (Kiesenwetter, 1844)

Examined material: 6 exs., Vădeni, 29.09.2010, bank of Prut River, area flooded in summer, leg. S.M.; 3 exs., Vlădeşti, bank of Prut River, 29.09.2010, leg. S.M.; 2 exs., Giurgiuleşti, 30.09.2010, area with temporary pools, a result of the summer flooding, leg. S.M.; 1 + , Movileni, pools with reed, 27.05.2009, leg. S.M.; 1 ex., Izvorul Dulce, 4.08.2005, leg. U.V.

Carpelimus rivularis (Motschulsky, 1860)

Examined material: 2 exs., Vădeni, 29.09.2010, bank of Prut River, area flooded in summer, leg. S.M.; 5 exs., Izvorul Dulce, 4.08.2005, leg. U.V.

Deleaster dichrous (Gravenhorst, 1802)

Examined material: 2 exs., Izvorul Dulce, 12.05.2007, at light trap, leg. U.V.

Oxytelus piceus (Linnaeus, 1767)

Examined material: 1 +, Fundu Mare Island, frequently flooded, 28.11.1994, leg. C.C.; 24 exs., Izvorul Dulce, 3.05.2005, on cattle fresh dung, bed of Slănic River, leg. U.V. 
Oxytelus sculptus Gravenhorst, 1806

Examined material: 1 ex., Izvorul Dulce, 13.09.2005, at light trap, leg. U.V.

Platystethus arenarius (Geoffroy, 1785)

Examined material: 1 ex., Izvorul Dulce, 3.05.2005, on cattle fresh dung, bed of Slănic River, leg. U.V.

Platystethus cornutus (Gravenhorst, 1802)

Examined material: $1 \hat{\jmath}$, Vădeni, 29.09.2010, area flooded in summer, leg. S.M.; 1 q, Movileni, pools with reed, 27.05.2009, leg. S.M.

Platystethus nitens (Sahlberg, 1832)

Examined material: 1 §̂, Gura Gârluţei, frequently flooded, 16.09.1994; 1 ex., Hogioaia Channel-entry in Stan's Marsh, frequently flooded, 3.10.1994; 1 ex., Fundu Mare Island, frequently flooded, 18.10.1994, leg. C.C.

Thinodromus dilatatus (Erichson, 1839)

Examined material: 2 exs., Vădeni, 29.09.2010, bank of Prut River, area flooded in summer, leg. S.M.

Subfamily Euaesthetinae Thomson, 1859

Euaesthetus bipunctatus (Ljungh, 1804)

Examined material: 1 क, Hogioaia Channel-entry in Stan's Marsh, area rarely flooded, 15.07.1994; $1 \hat{\delta}$, the same sampling place, in a frequently flooded area, 15.11.1994, leg. C.C.

Subfamily Steninae MacLeay, 1825

Stenus argus Gravenhorst, 1806

Examined material: 1 o, Rogojeni, Pochina Lake, wetland with Salix sp. and Rubus sp., 29.09.2010, leg. S.M.

Stenus biguttatus (Linnaeus, 1758)

Examined material: 2 ก, 1 q, Giurgiulești, 30.09.2010, area with temporary pools, leg. S.M.; 1 + , Salcia, sandy bank of Siret River, 27.05.2009; 3 o, 3 क, bank of Râmnicu Sărat River, Ciorăşti area, 27.05.2009, leg. S.M.; 1 ô, Bâscenii de Sus, Chiojd valley, 27.04. 2010, leg. S.M.; 2 §, 6 +, Valea Seaca, bank of Buzău River,

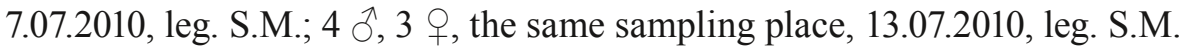

Stenus boops boops Ljungh, 1810

Examined material: 1 +, Vlădeşti, bank of Prut River, 29.09.2010, leg. S.M.

Stenus comma comma LeConte, 1863

Examined material: $4 \hat{\jmath}, 2$ o, Hogioaia Channel-entry in Stan's Marsh, area frequently flooded, 30.06 .1994 and 1 đે, 29.11.1994; 4 đ, Gura Gârluţei, frequently flooded, 29.11.1994; 1 ㅇ, 1 đ, Chirchineţu fish farm, frequently flooded, 29.11.1994, leg. C.C.

\section{Stenus humilis Erichson, 1839}

Examined material: 1 क, Hogioaia Channel-entry in Stan's Marsh, area rarely flooded, 30.07.1994; 2 , the same sampling place, rarely and frequently flooded areas, 1.08.1994; 10 , the same sampling place, 28.11.1994, leg. C.C. 
Stenus morio Gravenhorst, 1806

Examined material: 1 ex., Turcescu Islet, 28.08.2007, sandy bank of the Danube River, leg. S.M.; 2 ○, 1 q, Movileni, pools with reed, 27.05.2009, leg. S.M.

Subfamily Paederinae Fleming, 1821

Ochthephilum fracticorne (Paykull, 1800)

Examined material: 1 ex., Izvorul Dulce, 4.08.2005, leg. U.V.

Lathrobium pallidum Nordmann, 1837

Examined material: 2 , , Hogioaia Channel-entry in Stan's Marsh, area frequently flooded, 15.09.1994, leg. C.C.

Lathrobium taxi Bernhauer, 1902

Examined material: 1 ex., Izvorul Dulce, 4.05.2003, at light trap, leg. U.V.

Leptobium dimidiatum (Gridelli, 1926) (Fig. 2 E-H)

Examined material: Cama Islet: 2 o, 5 o , 29.05-3.06.2004 (collected from the Danube river bank on the base of a cliff-like high bank; river bank with wet, fine sized sand, without vegetation, temporarily flooded; river bank with muddy wet side, poor vegetation, poor in detritus); $3 \hat{\jmath}, 1$ क , 7-16.06.2004, leg. S.E. (the Danube river bank, forest with white willow, elm and tall herbaceous vegetation); 2 o, 1 q, 16.06.2004, leg. S.M. (the Danube river bank, in open area, from the mud from the edge of the temporary pools left behind the flooding); 10 , Vedea, 29.05-3.06.2004, leg. S.E. (in low hardwood forest with black poplar, oak, herbaceous layer and leaf litter); 3 ô, 3 o, Dinu Islet, 29.05-3.06.2004, leg. S.E. (hardwood forest with tall herbaceous vegetation; wet sandy river bank with some detritus); $1 \hat{\partial}$, Malu, 27.04.2004, leg. S.M. (grassland, pig dung).

Remarks: The specimens which were previously recorded under the name Leptobium gracile (Grav.) (Stan, 2005) were misidentified and belong to Leptobium dimidiatum. This species is highly similar to Leptobium gracile, but readily distinguished based on the morphology of the aedeagus. It had been recorded only from three scattered localities: Romania (Comana), Georgia (Tbilisi, Kumisi), Turkmenistan (Ashkhabad) (Assing, 2005).

\section{Paederus fuscipes fuscipes Curtis, 1826}

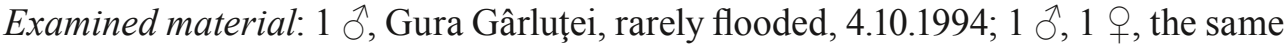
place, 28.11.1994; 2 đ, 1 \&, Chirchineţu fish farm, temporarily flooded, 30.10.1994; 7 exs., Movileni, pools with reed, 27.05.2009, leg. S.M.

Paederus littoralis littoralis Gravenhorst, 1802

Examined material: 1 ex., Izvorul Dulce, 9.06.2002; 1 ex., Cernăteşti, 31.08.2005, leg. U.V.

Rugilus orbiculatus (Paykull, 1789)

Examined material: 1 ex., Izvorul Dulce, 10.04.2002, leg. U.V.

Rugilus subtilis (Erichson, 1840)

Examined material: 1 ex., Izvorul Dulce, 10.04.2002; 1 ex., the same sampling place, 12.04.2002, leg. U.V. 
Scopaeus debilis Hochhuth, 1851

Examined material: 5 exs., Izvorul Dulce, 4.08.2005, at light trap, leg. U.V.

Scopaeus laevigatus (Gyllenhal, 1827)

Examined material: 2 exs., Izvorul Dulce, 4.08.2005, leg. U.V.

Sunius fallax (Lokay, 1919)

Examined material: 1 q, Hogioaia Channel-entry in Stan's Marsh, area rarely flooded, 15.07.1994, leg. C.C.; 1 †, bank of Râmnicu Sărat River, Ciorăşti area, 27.05.2009, leg. S.M.

Subfamily Staphylininae Latreille, 1802

Creophilus maxillosus maxillosus (Linnaeus, 1758)

Examined material: 1 ex., Izvorul Dulce, 30.04.2005, on carcasses, bed of Slănic River, leg. U.V.

Gabrius osseticus (Kolenati, 1846)

Examined material: 1 +, Gura Gârluţei, temporarily flooded, 28.11.1994, leg. C.C. Gauropterus fulgidus (Fabricius, 1787)

Examined material: 1 ex., Izvorul Dulce, 12.04.2002, leg. U.V.

Gyrohypnus fracticornis (Müller, 1776)

Examined material: 1 ex., Izvorul Dulce, 12.04.2002, leg. U.V.

Neobisnius procerulus procerulus (Gravenhorst, 1806)

Examined material: 1 + , Hogioaia Channel-entry in Stan's Marsh, area rarely flooded, 15.07.1994, leg. C.C.; 3 q, Rogojeni, Pochina Lake, wetland with Salix sp. and Rubus sp., 29.09.2010, leg. S.M.; 1 q, bank of Râmnicu Sărat River, Ciorăşti area, 27.05.2009, leg. S.M.

Ocypus nitens nitens (Schrank, 1781)

Examined material: 1 ex., Izvorul Dulce, 12.04.2002; 1 ex., the same sampling place, 18.04.2002; 1 ex., Unguriu, 28.04.2007 (in clearing, under stone), leg. U.V.

Ocypus ophthalmicus ophthalmicus (Scopoli, 1763)

Examined material: 1 ex., Izvorul Dulce, 3.05.2005, bed of Slănic River, leg. U.V.

Ontholestes murinus (Linnaeus, 1758)

Examined material: 3 exs., Izvorul Dulce, 3.05.2005, on cattle fresh dung, bed of Slănic River; 1 ex., the same sampling place, 16.04.2005, cattle dung, leg. U.V.

Philonthus cochleatus Scheerpeltz, 1937

Examined material: 4 exs., Izvorul Dulce, 3.05.2005, leg. U.V.

Philonthus coprophilus Jarrige, 1949

Examined material: 2 đે, 1 +, bank of Râmnicu Sărat River, Ciorăşti area, 27.05.2009, cattle dung, leg. S.M.

Philonthus cruentatus (Gmelin, 1790)

Examined material: 4 exs., Izvorul Dulce, 3.05.2005, on cattle fresh dung, bed of Slănic River, leg. U.V.

Philonthus diversiceps Bernhauer, 1901

Examined material: 1 ex., Movileni, pools with reed, 27.05.2009, leg. S.M.; 2 q, 2 के, Vădeni, 29.09.2010, bank of Prut River, area flooded in summer, leg. S.M. 
Philonthus ebeninus (Gravenhorst, 1802)

Examined material: 1 ex., Izvorul Dulce, 3.05.2005, on cattle fresh dung, bed of Slănic River leg. U.V.

Philonthus intermedius (Lacordaire, 1835)

Examined material: 2 9, bank of Râmnicu Sărat River, Ciorăşti area, 27.05.2009, cattle dung, leg. S.M.

Philonthus laminatus (Creutzer, 1799)

Examined material: 1 ex., Izvorul Dulce, 16.06.2002, leg. U.V.

Philonthus longicornis Stephens, 1832

Examined material: 1 Oె, bank of Râmnicu Sărat River, Ciorăşti area, 27.05.2009, cattle dung, leg. S.M.

Philonthus micans (Gravenhorst, 1802)

Examined material: 2 + , 2 ○, Rogojeni, Pochina Lake, wetland with Salix sp. and Rubus sp., 29.09.2010, leg. S.M.

Philonthus parvicornis (Gravenhorst, 1802)

Examined material: 3 exs., Izvorul Dulce, 16.04.2005, cow dung; 10 exs., the same sampling place, 3.05.2005, on cattle fresh dung, bed of Slănic River, leg. U.V.

Philonthus pseudovarians Strand, 1941

Examined material: 1 ex., Izvorul Dulce, 3.05.2005, on fresh dung, bed of Slănic River, leg. U.V.

Philonthus punctus punctus (Gravenhorst, 1802)

Examined material: 1 \&, 1 J, Vădeni, 29.09.2010, bank of Prut River, area flooded in summer, leg. S.M.; 2 +, Giurgiuleşti, 30.09.2010, area with temporary pools, leg. S.M.

Philonthus quisquiliarius quisquiliarius (Gyllenhal, 1810)

Examined material: 2 ô, 1 \&, Giurgiuleşti, 30.09.2010, area with temporary pools, leg. S.M.; 1 +, 1 đ̊, Vădeni, 29.09.2010, bank of Prut River, area flooded in summer, leg. S.M., 7 exs., Izvorul Dulce, 4.08.2005, leg. U.V.

Philonthus salinus Kiesenwetter, 1844

Examined material: 1 đૈ, Gura Gârluţei, temporarily flooded, 28.11.1994, leg. C.C.

Philonthus spinipes spinipes Sharp, 1874

Examined material: 1 ex., Izvorul Dulce, 25.07.2002, cattle fresh dung; 2 exs., the same sampling place, 9.05.2004, under stones on the bank of Slănic River; 1 ex., Berca, 1.09.2005, horse dung, leg. U.V.

Platydracus stercorarius stercorarius (Olivier, 1795)

Examined material: 1 ex., Unguriu, 28.04.2007, leg. U.V.

Stenistoderus nothus (Erichson, 1839)

Examined material: 1 ex., Chirchineţu fish farm, area temporarily flooded, 29.11.1994, leg. C.C. 


\section{Xantholinus dvoraki Coiffait, 1956}

Examined material: Hogioaia Channel-entry in Stan's Marsh: 4 q, temporarily flooded, 30.06.1994, 1 क, rarely flooded area, 17.07.1994; 2 q, temporarily flooded, 2.09.1994; 3 त, 1 q, frequently flooded, 16.11.1994; 12 exs., temporarily flooded, 28.11.1994; 16 ô, 4 , frequently flooded, 28.11.1994; 2 o, 3 क, rarely

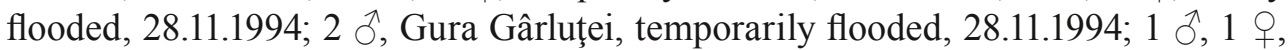
Chirchineţu fish farm, 28.11.1994; 1 $\curvearrowright, 1$ q, Fundu Mare Island, temporarily flooded area, 28.11.1994, $1 \hat{\delta}, 1$ o, the same site, frequently flooded, 28.11.1994, leg. C.C.

The results represent a part of the Bilateral Collaboration Project within the Agreement between the Romanian Academy and the National Academy of Sciences of Belarus: "Distribution patterns of staphylinid species (Coleoptera: Staphylinidae) from the riparian ecosystems of East Europe (south-north direction) - case study Romania and Belarus".

\section{ACKNOWLEDGEMENTS}

The author wants to thank to Mr László Ádám (Budapest, Hungary) for the identification of the species listed in this paper. Also, thanks to all colleagues who donated the collected material and which was added to the staphylinid collection of the museum: Dr. Constantin Ciubuc, Dr. Eckbert Schneider and Viorel Ungureanu. Thanks to Dr. Alexandru Iftime for making the map with the sampling sites, using the software Arc View GIS version 8.3. Moreover, thanks are extended Dr. Costică Adam for the photos of Thecturota marchii made with an Olympus BX 41 microscope and to my colleague George Năzăreanu for the photos of Leptobium dimidiatum. I also thanks the referees for their constructive comments on my manuscript and for their publishing advice.

\section{CONTRIBUȚII LA CUNOAŞTEREA STAFILINIDELOR (COLEOPTERA: STAPHYLINIDAE) DIN CÂTEVA ECOSISTEME RIPARIENE DIN ZONA DE SUD-EST A ROMÂNIEI}

\section{REZUMAT}

Diversitatea faunei de stafilinide este investigată în câteva ecosisteme ripariene prezente de-a lungul unor râuri din zona de sud - est a României: Dunăre, Prut, Siret, Buzău. 94 specii şi subspecii de stafilinide au fost identificate în 23 de situri investigate. Thecturota marchii (Dodero) este o semnalare nouă în fauna României. Leptobium dimidiatum (Grideli), o specie rară, este semnalată dintr-un nou sit, al doilea pe teritoriul României. Pentru fiecare specie se prezintă situl de colectare, data, numărul de exemplare (sexul pentru cele mai multe), colectorul.

\section{LITERATURE CITED}

ASSING, V., 2005 - A revision of the genus Leptobium Casey (Coleoptera: Staphylinidae: Paederinae). Stuttgarter Beiträge zur Naturkunde, Serie A (Biologie), 673: 1-182.

HORION, AD., 1967 - Faunistik der Mitteleuropäischen Käfer. Staphylinidae. 3. Habrocerinae bis Aleocharinae (Ohne Subtribus Athetae). 11: xxiv + 1-419. Überlingen-Bodensee: P. C. W. Schmidt.

SMETANA, A., 2004 - Staphylinidae. In: I. Löbl, A. Smetana (eds), Catalogue of Palaearctic Coleoptera. II. Hydrophiloidea - Histeroidea -Staphylinoidea. Stenstrup, Apollo Books: $942 \mathrm{pp}$. 
STAN, M., 2005 - Rove beetles (Coleoptera: Staphylinidae) from the Danube floodplain area, Giurgiu sector (Romania). Travaux du Muséum National d'Histoire Naturelle "Grigore Antipa", 48: 87-101.

STAN, M., 2009 - Taxonomical diversity of terrestrial coleopterans (Insecta: Coleoptera) collected from eight Danube islets (Călăraşi county). Muzeul Olteniei Craiova. Oltenia. Studii şi comunicări. Ştiinţele Naturii, 25: 114-118.

Received: May 13, 2011

Accepted: November 29, 2011
Muzeul Naţional de Istorie Naturală

„Grigore Antipa”

Şos. Kiseleff, nr. 1, 011341 Bucureşti 2, România

e-mail:mstan@antipa.ro 